port of other photoexcited states in $\mathrm{Si}$ and Ge.

We wish to thank E. E. Haller for supplying the Si crystal, and M. W. Klein for the loan of the special photomultiplier tube. This project was supported by the National Science Foundation under the Materials Research Laboratory Grant DMR-77-23999, and by a Cottrell equipment grant.

${ }^{1}$ R. Haynes and W. Shockley, Phys. Rev. 75, 691L (1949). See also W. Shockley, Holes and Electrons in Semiconductors (Van Nostrand, New York, 1950).

${ }^{2}$ G. L. Pearson and J. Bardeen, Phys. Rev. $\underline{75}, 865$ (1949) .

${ }^{3}$ P. P. Debye and E. M. Conwell, Phys. Rev. $\underline{93}$,
693 (1954).

${ }^{4}$ M. Costato and S. Scavo, Nuovo Cimento 54B, 169 (1968).

${ }^{5}$ R. S. Markiewicz, J. P. Wolfe, and C. D. Jeffries, Phys. Rev. B 15, 1988 (1977).

${ }^{6}$ P. L. Gourley and J. P. Wolfe, Phys. Rev. Lett. $\underline{40}$, 526 (1978).

${ }^{7}$ A. Varian, VPM-164 cooled to $200 \mathrm{~K}$.

${ }^{8}$ J. M. Ziman, Electrons and Phonons (Oxford Univ. Press, Oxford, 1960), p. 433.

${ }^{9} \mathrm{As}$ long as $\lambda_{p h}>a_{0}$, where $a_{0}$ is the exciton Bohr radius, the exciton deformation potential is taken to be the shift in the band-gap energy with strain. This shift has been directly measured by I. Balslev, Phys. Rev. 143,636 (1966).

${ }^{10} \mathrm{~J}$. C. Hensel, Phys. Lett. 4 , 38 (1963).

${ }^{11}$ E. Otsuka, T. Ohyama, and K. Murase, J. Phys. Soc. Jpn. 25, 729 (1968).

\title{
Quantum Magnetic Hamiltonians with Remarkable Spectral Properties
}

\author{
Keith Miller ${ }^{(a)}$ and Barry Simon ${ }^{(b)}$ \\ Department of Physics, Princeton University, Princeton, New Jersey 08544 \\ (Received 3 March 1980)
}

\begin{abstract}
The Hamiltonian, $H$, of a spinless particle moving in two dimensions in an axially symmetric magnetic field $B(\rho)$ is considered. If $B(\rho) \sim \rho^{-\alpha}$ for $\rho$ large with $0<\alpha<1$, then it is shown that $H$ has spectrum $[0, \infty)$ with only eigenvectors and eigenvalues dense in $[0, \infty)$. If $\alpha=1$, then the spectrum is a dense point spectrum in $[0, c]$ for suitable $c$ and absolutely continuous in $[c, \infty)$.
\end{abstract}

PACS numbers: $71.55 . \mathrm{Jv}, 71.30 .+\mathrm{h}$

A subject of current interest is the study of quantum Hamiltonians describing electrons in random potentials. In terms of the spectrum, the conventional wisdom ${ }^{1}$ is that in one dimension, $H$, after adding a suitable constant, will have the following properties (Type I): The allowed energy values (spectrum) are $[0, \infty)$ but there is a complete set of eigenvectors. This is accomplished by having eigenvalues $\lambda_{n}$ which are dense in $[0, \infty)$.

In more than one dimension, the expectation is Type II: The spectrum is still $[0, \infty)$. There is a number $d$ (the "mobility edge") with dense point spectrum in $[0, d]$ and with absolutely continuous spectrum in $[d, \infty)$.

Until one is used to it, these spectral properties are rather surprising, in part because one cannot write down an explicit "random" potential. For this reason, there may be some point in finding explicit Hamiltonians with these properties. We emphasize that no relation is claimed between the physical reasons for the spectral types found below and those in random systems. These results are, at this point, something of curiosities, but may have some relevance to physics in suitable magnetic fields. There also may be some point in studying transport at the mobility edge in these problems.

To begin with some explicit examples, let $\rho$ $=\left(x^{2}+y^{2}\right)^{1 / 2}$. Then, the combination of operators

$$
\left(\frac{1}{i} \frac{\partial}{\partial x}+\frac{c y}{(1+\rho)^{\alpha}}\right)^{2}+\left(\frac{1}{i} \frac{\partial}{\partial y}-\frac{c x}{(1+\rho)^{\alpha}}\right)^{2}
$$

has type-I spectral properties ${ }^{2}$ if $0<\alpha<1$, typeII with $d=c^{2}$ if $\alpha=1$. For $\alpha>1$, the spectrum is purely absolutely continuous.

The point of the proof of these properties is to note that (1) is the Hamiltonian of a spinless particle in two dimensions moving in a magnetic field, so that different spectral properties can be obtained by working in different gauges. 


\section{Let}

$$
H(a)=\left(-i \partial / \partial x-a_{x}\right)^{2}+\left(-i \partial / \partial y-a_{y}\right)^{2},
$$

and let $B=\nabla \times \vec{a} \equiv \partial_{x} a_{y}-\partial_{y} a_{x}$. We first assert that if $B \rightarrow 0$ at infinity, then ${ }^{3}$ the spectrum of $H(\vec{a})$ is $[0, \infty)$. To prove this we first note Weyl's criterion ${ }^{4}$ for the spectrum: $E \in \sigma(H(\vec{a}))$ if and only if there exists a sequence $\varphi_{n}$ of vectors with

$$
\left\|[H(\vec{a})-E] \varphi_{n}\right\| \rightarrow 0 .
$$

If $\nabla \times \overrightarrow{\mathrm{a}}^{\prime}=B$ also, then $e^{i \lambda} H\left(\overrightarrow{\mathrm{a}}^{\prime}\right) e^{-i \lambda}=H(\overrightarrow{\mathrm{a}})$ for suitable $\lambda$. Thus, for (3) to hold, it suffices to find a gauge, ${ }^{3} \overrightarrow{\mathrm{a}}^{\prime}$, with

$$
\left\|\left[H\left(\vec{a}^{\prime}\right)-E\right] \psi_{n}\right\| \rightarrow 0 ;
$$

one need only take $\varphi_{n}=e^{+i \lambda} \psi_{n}$. We will take the gauge

$$
a^{\prime}{ }_{y}(x, y)=\int_{0}^{x} B(s, y) d s ; a^{\prime}{ }_{x} \equiv 0 .
$$

Since $B \rightarrow 0$ at infinity we can find a sequence of numbers $r_{n}$ so that everywhere in the square of side $n$ side centered about $\left(0, r_{n}\right)$, one has $\mid \vec{a}^{\prime}(x$, $y) \mid \leqslant 2^{-n}$. Take a fixed smooth function, $\eta$, vanishing outside the unit square with $L^{2}$-norm 1 , fix $k$, and let

$$
\psi_{n}(x, y)=e^{i k x} n^{-1} \eta(x / n, y-r n / n) .
$$

Then (4) holds with $E=k^{2}$. Since $k$ is arbitrary, the spectrum of $H(a)$ contains $[0, \infty)$ if $B \rightarrow 0$ at infinity. Since $H(\vec{a}) \geqslant 0$, the spectrum equals $[0, \infty)$.

To analyze the spectrum further, we suppose that $B$ is axially symmetric, i.e., $B(x, y)=b(\rho)$. Pick the radial gauge

$$
a_{x}(x, y)=y \rho^{-1} a(\rho), \quad a_{y}(x, y)=-x \rho^{-1} a(\rho),
$$

with

$$
a(\rho)=(2 \rho)^{-1} \int_{0}^{\rho} s b(s) d s .
$$

In this gauge, $H(a)$ commutes with $L_{z}=i^{-1} \partial / \partial \varphi$ and, restricted to functions with $L_{\boldsymbol{z}}=m$ (indicated by the symbol $\uparrow$ ),

$$
H(a)\left\lceil\left(L_{\boldsymbol{z}}=m\right)=-\Delta_{\rho}+m^{2} \rho^{-2}-2 m \rho^{-1} a+a^{2} .\right.
$$

This operator is the same as the $\left(L_{z}=0\right.$ operator for the potential problem with potential

$$
V=m^{2} \rho^{-2}-2 m \rho^{-1} a+a^{2} .
$$

Since $b \rightarrow 0$ at infinity, $\rho^{-1} a \rightarrow 0$ at infinity. If $s b(s)$ $\rightarrow \infty$ at infinity, then $a \rightarrow \infty$ at infinity so that $V$ $\rightarrow \infty$ at infinity and hence each $H(a) \uparrow\left(L_{z}=m\right)$ has point spectrum, indeed ${ }^{4}$ localized eigenvectors with exponential falloff. Thus the spectrum $H(a)$ is pure point but of necessity dense. We summarize with Theorem 1: If $b \rightarrow 0$ at infinity but $\rho b(\rho) \rightarrow \infty$ at infinity, then $H(a)$ has type-I spectrum.

Next suppose that

$$
b(\rho)=c_{0} \rho^{-1}+d_{0} \rho^{-2}+O\left(\rho^{-2-\epsilon}\right) .
$$

Then

$$
a(\rho)=\frac{1}{2} c_{0}+\frac{1}{2} \rho^{-1} \ln \rho+O\left(\rho^{-1-\epsilon}\right),
$$

so that

$$
V=\frac{1}{4} c_{0}^{2}+\frac{1}{2} c_{0} \rho^{-1} \ln \rho-m \rho^{-1} c_{0}+O\left(\rho^{-1-\epsilon}\right) .
$$

For such potentials, one knows ${ }^{6}$ that $-\Delta_{\rho}+V$ has discrete spectrum in $\left(-\infty, \frac{1}{4} c_{0}^{2}\right)$ and absolutely continuous (Coulomb-type scattering) states in $\left[\frac{1}{4} c_{0}{ }^{2}, \infty\right)$, so that (Theorem 2) if (5) holds, then $H$ has type-II spectrum with $d=\frac{1}{4} c_{0}{ }^{2}$. Similar arguments show that (Theorem 3) if $b(\rho)=c \rho^{-\alpha}$ $+O\left(\rho^{-\alpha-1}\right)$ with $\alpha>1$, then $H$ has purely absolutely continuous spectrum

Some insight is obtained by noting that a classical particle moving in an axially symmetric magnetic field obeying Eq. (5) will be able to escape to infinity rather than be trapped by the field if and only if the energy is larger than $\frac{1}{4} c_{0}^{2}$. Indeed, this follows easily by looking at conservation of energy and angular momentum as in the quantum case.

It is a pleasure to thank J. Hopfield and V. Enss for emphasizing to us that we should look at the classical case. This research was supported in part by the National Science Foundation under Grant No. MCS-78-01885.

\footnotetext{
(a) National Science Foundation predoctoral fellow.

(b) Also at Department of Mathematics, Princeton University, Princeton, N. J. 08544.

${ }^{1}$ There are now rigorous proofs of the one-dimen-
} sional result for suitable random potentials; see I. Gol'dsheid, S. A. Molchanov, and L. Pastur, Funkts. Anal. Prilo. 11, 1 (1977) [Funct. Anal. Appl. 11, 1 (1977)].

${ }^{2} J$. Avron, I. Herbst, and B. Simon, Duke Math. J. 45,847 (1978), already noted that when $0<\alpha<1$, there is a complete set of normalizable eigenstates but they did not note that $\sigma(H)=[0, \infty)$ and thus the denseness of the eigenvalues.

${ }^{3}$ A similar argument with an $n$-dependent gauge proves the same result in three dimensions; see K. Miller, unpublished.

${ }^{4}$ M. Reed and B. Simon, in Methods of Modern Mathe matical Physics (Academic, New York, 1972 and 1978), Vols. 1 and 4.

${ }^{5}$ For simplicity we suppose that $\vec{a}$ and $\vec{a}^{\prime}$ are smooth.

${ }^{6}$ See, e.g., R. Lavine, J. Funct. Anal. 12, 30-54 (1973). 